\title{
Computational Analysis of Electron Transpiration Cooling for Hypersonic Vehicles
}

\author{
Kyle M. Hanquist* and Iain D. Boyd ${ }^{\dagger}$ \\ Department of Aerospace Engineering, University of Michigan, Ann Arbor, MI
}

\begin{abstract}
Simulations of a leading edge of a hypersonic vehicle using computational fluid dynamics (CFD) and a material response code are presented in order to investigate the effect in-depth surface conduction has on electron transpiration cooling (ETC). ETC is a recently proposed thermal management approach. Previous numerical studies have shown that ETC can significantly lower the stagnation point surface temperature of sharp leading edges of hypersonic vehicles. However, these studies have neglected the effect of heat also being conducted into the material as opposed to only into the flow via radiative cooling and ETC. A modeling approach is presented for ETC, which includes the boundary conditions for electron emission from the surface, accounting for the electric field and space-charge limit effects within the near-wall plasma sheath. A material response code is used to determine typical values of in-depth surface conduction for the test cases studied. Since ETC materials are still being developed, a parametric study is conducted for a range of material properties pertinent to ETC. The results of this study are used to generate in-depth surface conduction profiles, which are implemented into the CFD framework. The CFD simulations show that including in-depth surface conduction results in lower surface temperatures than predicted with radiative and ETC cooling alone. This is because in-depth surface conduction complements radiative cooling and ETC by moving heat away from the surface, in the case of surface conduction by moving the energy into the material, allowing for a lower surface temperature. The results also show that ETC remains a major mode of heat transfer away from the surface, even with in-depth surface conduction. This suggests that ETC is still a promising mode of thermal management, especially since it transfers energy to the flow instead of into the material.
\end{abstract}

\section{Nomenclature}

$A_{R} \quad$ Richardson constant, $1.20 \times 10^{6} \mathrm{~A} / \mathrm{m}^{2} / \mathrm{K}^{2}$

$C_{i} \quad$ Ion acoustic speed

$D \quad$ Diffusion coefficient

D Drag

E Electric field

j Electric current density

$J \quad$ Current density

$k_{B} \quad$ Boltzmann constant, $1.38 \times 10^{-23} \mathrm{~J} / \mathrm{K}$

$\dot{m} \quad$ Mass blowing rate

$M_{s} \quad$ Molar mass of species $s$

$n \quad$ Number density

$N_{A v}$ Avogadro constant, $6.02 \times 10^{23} \mathrm{~mol}^{-1}$

$p \quad$ Pressure

$q \quad$ Heat transfer

$Q_{e} \quad$ Elementary charge, $1.60 \times 10^{-19} \mathrm{C}$

*PhD Candidate, Student Member AIAA.

$\dagger$ James E. Knott Professor of Engineering, Fellow AIAA 


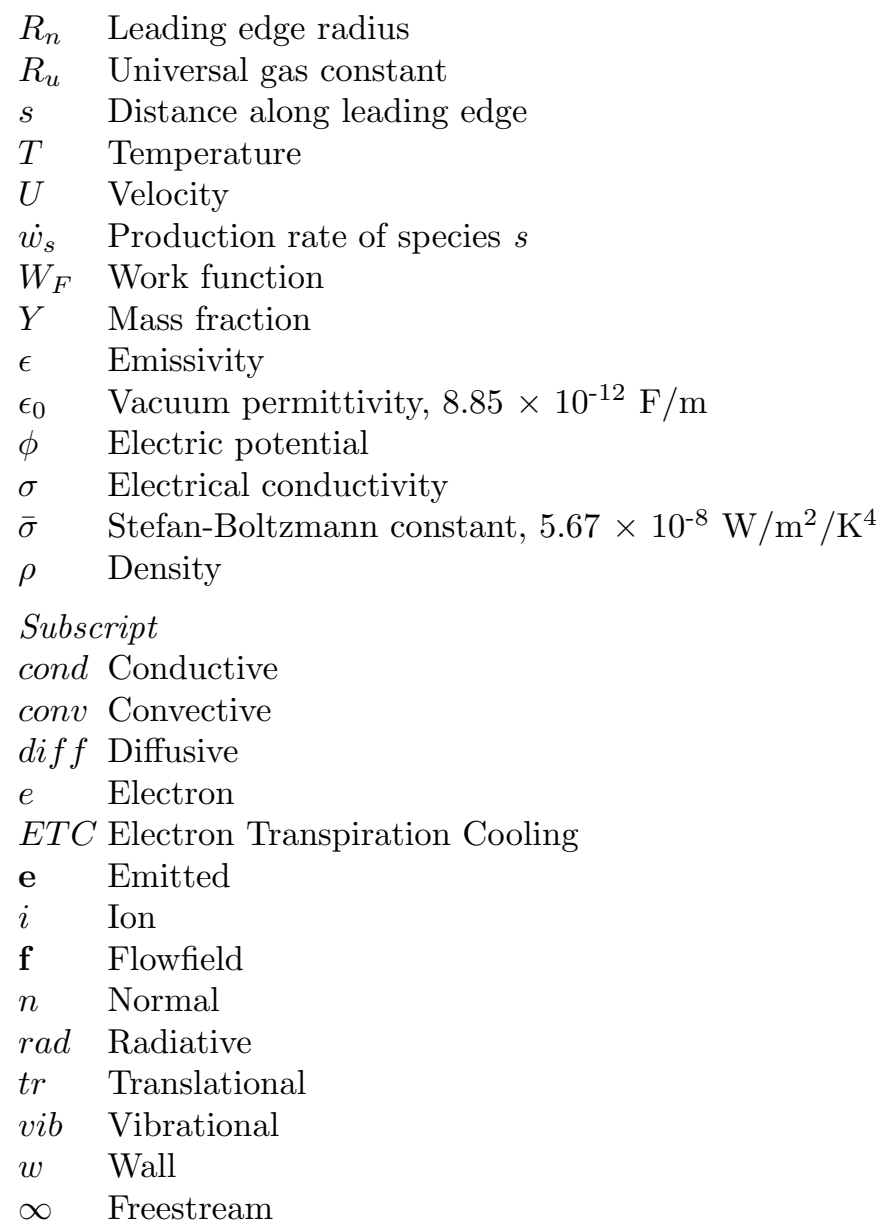

\section{Introduction}

NE of the fundamental challenges of hypersonic flight is the management of the high heat loads inherent to high-speed flight. ${ }^{1}$ In order to maximize the range of any flight vehicle, the drag must be minimized, which essentially is designing a slender body with sharp leading edges for the case of hypersonic flight vehicles. This is shown by a theoretical analysis performed by Lees and Kubota, ${ }^{2}$ which revealed the drag is proportional to the leading edge radius,

$$
\mathbf{D} \propto(1 / 2) \rho_{\infty} U_{\infty} R_{n}^{k+1}
$$

where $k=0$ for a two-dimensional body and $k=1$ for an axisymmetric body. However, this decrease in drag comes at a cost of increased convective heat transfer as shown by a later theoretical analysis performed by Fay and Riddell, ${ }^{3}$ which shows that the stagnation point heat transfer is inversely proportional to the square-root of the vehicle leading edge radius,

$$
q_{\text {conv }} \propto \sqrt{\frac{\rho_{\infty}}{R_{n}}} U_{\infty}^{3}
$$

Equations 1 and 2 show that minimizing a property such as freestream density can reduce the drag while also lowering the convective heating, which is why hypersonic flight often occurs at high altitudes. However, if the leading edge radius is reduced to limit the drag, there is a corresponding increase in convective heat transfer. Given that reducing the drag is generally one of the leading design criteria for flight especially at high speeds, high convective heating rates will be inherent and managing them will be vital. Currently, there are multiple approaches to manage the heat loads, which are discussed in detail in Ref. 4. One approach is to have a leading edge material able to withstand high-temperatures without degrading. One type of material that meets this criteria are ultra-high temperature composite (UHTC) materials, which were used on the 
NASA X-43 experimental hypersonic aircraft. ${ }^{5}$ Since these materials can withstand high temperatures, the high convective heat rates are managed by radiative cooling, which is determined by Stefan-Boltzmann law,

$$
q_{\text {rad }}=\epsilon \bar{\sigma} T_{w}^{4}
$$

Since radiative cooling has a fourth power relation to the surface temperature, having a material that can withstand high surface temperatures is highly beneficial. Other modes of heat management include more of an active approach such as ablation. Ablation has significant heat management benefits through essentially a controlled thermo-chemical decomposition of the heat shield, which makes it a great approach for reentry flight. This shape change of the surface, while permitted for the blunt bodies of re-entry flight, is unacceptable for the sharp leading edges of hypersonic vehicles. An alternative approach that has been proposed involves using thermo-electric materials at the leading edges of hypersonic vehicles and is called Electron Transpiration Cooling (ETC). ${ }^{6}$ When exposed to high surface temperatures experienced during the extreme convective heating rates, these materials emit a current of electrons that may lead to a transpiration cooling effect of the surface of the vehicle. This phenomenon is known as thermionic emission and occurs when the thermal energy given to the electrons is greater than the binding potential of the surface material. A recent numerical study was completed and showed that ETC can reduce the stagnation point surface temperature by $11 \%$ and $50 \%$ for a $6 \mathrm{~km} / \mathrm{s}$ and $8 \mathrm{~km} / \mathrm{s}$ test case, respectively, at $60 \mathrm{~km}$ altitude. ${ }^{7}$ This study, however, neglected the effect of in-depth surface conduction.

With all of these approaches, a portion of the heat will conduct into the material, to some extent. Allowing the heat to conduct into the material and then manage it with cooling pipes is another mode to manage the heating loads, although the sharp leading edge radii limit the space for cooling pipes. Essentially, the heat transfer at the leading edge of the vehicle is,

$$
q_{\text {in }}=q_{\text {away }}
$$

where the heat transfer into the surface is the convective heat transfer, which will be defined as the sum of the conductive heat flux (temperature gradients in the flow) and diffusive (species gradients in the flow) heat flux,

$$
q_{\text {in }}=q_{c o n v}=q_{c o n d, f l}+q_{d i f f}
$$

Radiative heating is not considered because it is typically negligible compared to convective heating for the very slender bodies (e.g. leading edges) used in hypersonic flight. ${ }^{8}$ The heat away from the surface will be defined as either heat distributed back out into the flow typically through radiative or also ETC in this study, or heat conducted into the material through in-depth surface conduction,

$$
\begin{gathered}
q_{\text {away }}=q_{\text {out }}+q_{\text {in }} \\
q_{\text {out }}=q_{\text {rad }}+q_{E T C}
\end{gathered}
$$

The surface temperature is determined based on this balance of heat transfer. In order to assess whether ETC is a viable method of thermal load management, in-depth surface conduction must also be included in the analysis, which is the focus of the paper. First, the numerical methods are discussed that are used to model the hypersonic flow and material response along with the test case description for the simulations. Then, numerical results are presented for in-depth surface conduction for the test cases without ETC. Profiles for in-depth surface conduction are then generated from this study and implemented into the ETC modeling framework. The results are presented that highlight the effect in-depth surface conduction has on the benefits of ETC, specifically surface temperature. Finally, the paper presents some conclusions drawn from this study and outlines future work recommendations.

\section{Numerical Approaches}

\section{A. Fluid}

The flow is modeled using computational fluid dynamics (CFD) using a code developed at the University of Michigan called LeMANS. ${ }^{9}$ LeMANS is a parallel, three-dimensional code that solves the Navier-Stokes equations on unstructured computational grids. LeMANS includes thermo-chemical non-equilibrium effects and the flow is modeled assuming that the continuum approximation is valid. It is also assumed that the translational and rotational energy modes can be described by a single temperature, $T_{t r}$, and that the vibrational, electronic, and electron translational energy modes are described by a different temperature, $T_{v i b}$. The CFD code is discussed in detail in Refs. 9 and 10. 


\section{Electron Emission}

The electron current density is a function of the material's surface temperature and work function as determined by Richardson, ${ }^{11}$

$$
J_{e, \mathbf{e}}=A_{R} T_{w}^{2} \exp \left(\frac{-W_{F} Q_{e}}{k_{B} T_{w}}\right)
$$

It is to be noted that this current is only realized in ideal conditions: emitted electrons see no retarding electric field at the surface, are not reflected back to the surface through collisions, nor see a virtual cathode created by space-charge limits. These space-charge limits are discussed in the following sections. A boundary condition is implemented into LeMANS to model the production rate of electrons,

$$
\dot{w}_{e}=\frac{J_{e, \mathbf{e}}}{Q_{e} N_{A v}}-\sum_{i=\text { ions }} \dot{w}_{i} .
$$

A quantity of the emitted electrons and flowfield ions will recombine at the surface and is determined by assuming that the surface is fully catalytic to ions: ${ }^{12}$

$$
\dot{w}_{i}=\frac{\rho_{i}}{M_{i}} \sqrt{\frac{R_{u} T_{w}}{2 \pi M_{i}}}
$$

The gas properties at the surface of the hypersonic vehicle are calculated in LeMANS by solving the species mass balance,

$$
\rho_{w} D_{s, w} \nabla Y_{s, w}+\dot{m} Y_{s, w}=M_{s} \dot{w}_{s}
$$

and momentum balance,

$$
p_{n}+\rho_{n} u_{n}^{2}=p_{w}+\frac{\dot{m}^{2}}{\rho_{w}}
$$

equations to obtain the gas density, species mass fractions, and gas normal velocity. The mass blowing rate is defined as the product of the electron production rate and the electron mass,

$$
\dot{m}=m_{e} \dot{w}_{e} .
$$

The emitted electrons will carry away energy from the vehicle surface at a flux of: ${ }^{11}$

$$
q_{E T C}=J_{e e}\left(W_{F}+\frac{2 k_{B} T_{w}}{Q_{e}}\right),
$$

which accounts for the energy the electrons use overcoming the potential barrier of the material work function as well as the kinetic energy associated with the emitted electrons.

\section{Electric Field}

Due to the emitted species possessing an electric charge, LeMANS is modified in order to model the electric field, to some extent. The electric field is determined by the gradient of the electric potential,

$$
\vec{E}=-\nabla \phi
$$

where the electric potential is calculated using Ohm's law and solving the steady state current continuity equation, ${ }^{13}$

$$
\begin{gathered}
\vec{j}=\sigma \vec{E} \\
\nabla \cdot \vec{j}=\nabla \cdot(\sigma \nabla \phi)=0
\end{gathered}
$$

The electrical conductivity is approximated semi-empirical model based on the flow temperature developed by Razier, ${ }^{14}$ which is valid for air, nitrogen, and argon,

$$
\sigma=8300 \times \exp \left(\frac{-36,000}{T}\right)[m h o / m] .
$$


The electric field at the wall can decrease the energy barrier that the emitted electrons must overcome at the surface, which effectively reduces the work function, known as the Schottky effect: ${ }^{15}$

$$
W_{F, c}=W_{F}-\sqrt{\frac{Q_{e}^{3} E_{w}}{4 \pi \epsilon_{0}}}
$$

Previous work showed that the magnitude of the electric field for cases similar to this study has a relatively small value, which causes negligible effects on the predicted surface properties of hypersonic vehicles with thermo-electric TPS materials, ${ }^{16}$ but will play an important role in future work where electrons must reattach downstream on the aftbody to prevent the vehicle from accumulating a net charge.

\section{Plasma Sheath}

A plasma sheath forms near the the wall, which is a non-neutral region between the quasineutral flowfield and the wall. The sheath typically occurs because the electrons are much more mobile than the ions due to their mass difference. This higher mobility of electrons leads to more electrons leaving this region than ions, leaving the region positively charged, which generates a negative potential difference between the flowfield and the wall. Previous work showed that ETC is susceptible to space-charge limits in which a virtual cathode forms within the collisionless sheath pushing a portion of the electrons back to the surface before they escape into the hypersonic flow. ${ }^{7}$ This effect can limit the level of emission from the surface resulting in smaller emission currents than predicted by vacuum conditions of Eq. 8. Space-charge limited electron emission can be determined by, ${ }^{17}$

$$
J_{e, \mathbf{e}, s c}=\frac{Q_{e} n_{i} C_{i} G}{1+A \cdot G} \sqrt{\frac{-2 \Phi_{v c} m_{i}}{m_{e}}}
$$

where the surface bias, or surface potential, is normalized by the electron temperature,

$$
\Phi_{v c}=\frac{Q_{e}\left(\phi_{v c}-\phi_{0}\right)}{T_{e}}
$$

In this work, the value of the virtual cathode potential (or wall potential if the space-charge limit is not reached) is set to a value. The electron temperature is not calculated explicitly but is approximated using an approach developed in Ref. 7. The ion acoustic speed is defined by,

$$
C_{i}=\sqrt{\frac{k_{B} T_{e}}{m_{i}}} .
$$

$\mathrm{G}$ and $\mathrm{A}$ are functions of the normalized virtual cathode potential and the ratio of the emitted electron temperature (electrons are emitted at the material surface temperature) to the electron temperature at the sheath edge. If the current given by Eq. 20 is less than the current given by Eq. 8, emission is space-charge limited and Eq. 20 is used.

\section{B. Material}

The material is modeled using a material response solver developed at the University of Michigan called MOPAR. ${ }^{18}$ The numerical code is capable of simulating multidimensional thermal and structural response of materials exposed to hypersonic flows. The thermal portion of the code can model materials with temperature-dependent, anisotropic properties and the heat fluxes are computed using Fourier's law. MOPAR is strongly coupled with LeMANS in order to study quasi-static aerothermal and aerothermoelastic problems that arise in hypersonic flows such as ablation and in-depth surface conduction. The material response solver is discussed in detail in Refs. 18 and 19.

\section{Numerical Results}

\section{A. Test Case Description}

\section{Geometry}

The geometry of the test case is based on the IRV-2 vehicle nose shape. ${ }^{20}$ The nose radius of the geometry is $1.905 \mathrm{~cm}$ with a $8.42 \mathrm{deg}$. cone angle as shown in Figure 1. This geometry was used because it is typical 
of a sharp leading edge for a hypersonic vehicle and computational grids have already been generated for the flow and material, and the grids were verified in a previous study. ${ }^{18}$ Previous ETC studies have used a 2D planer wedge with a $1 \mathrm{~cm}$ leading edge radius and $5 \mathrm{deg}$. wedge angle so the grids are scaled down to match a $1 \mathrm{~cm}$ nose radius and will be used as an 2D planar wedge instead of an axisymmetric cone. The computational domain and boundary conditions are shown in Fig. 2.

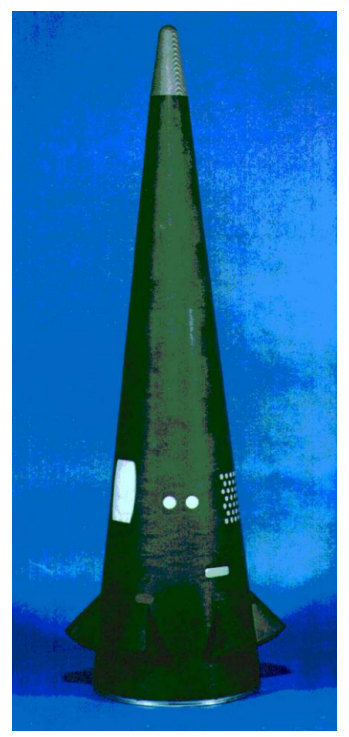

Figure 1: IRV-2 vehicle geometry with nose shown in grey [Ref. 20]

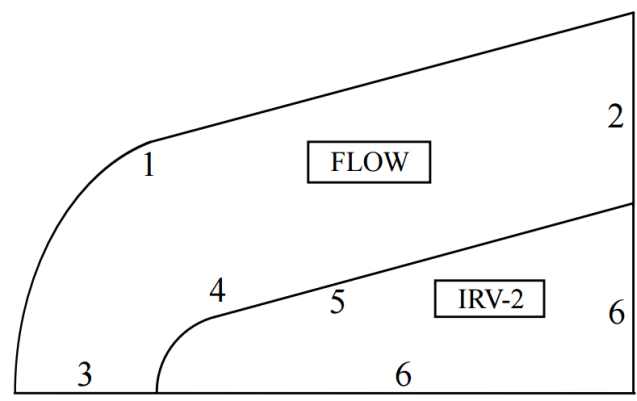

1. Supersonic inlet

2. Supersonic outlet

3. Symmetry

4. Coupled, no-slip wall

5. Aerodynamic heating

6. Adiabatic

Figure 2: The computational domain and boundary conditions for simulations [Ref. 18]

\section{Flow Conditions}

The freestream flow conditions correspond to conditions at $60 \mathrm{~km}$ altitude and are summarized in Table 1. Although the freestream primarily consists of two species, post-shock chemistry will generate more species in which the numerical approach accounts for 11-species air. The freestream velocity in the test cases will be $6 \mathrm{~km} / \mathrm{s}$, unless otherwise noted. This velocity was chosen based on a previous study that showed that ETC is only beneficial for high freestream velocities, whereas slower freestream velocities are greatly limited by space-charge effects. ${ }^{21}$ Previous studies of ETC have been at steady-state, whereas now with the addition of in-depth surface conduction, the duration of the test case is influential on the results. Three times of duration are investigated include one, five, and ten minute durations. 
Table 1: Freestream properties

\begin{tabular}{ccc}
\hline Temperature $[\mathrm{K}]$ & \multicolumn{2}{c}{ Density $\left[\mathrm{kg} / \mathrm{m}^{3}\right]$} \\
& $\mathrm{N}_{2}$ & $\mathrm{O}_{2}$ \\
\hline 238 & $1.76 \times 10^{-4}$ & $5.39 \times 10^{-5}$ \\
\hline
\end{tabular}

\section{Material Conditions}

Since the material for ETC is currently being developed many of it's material properties are unknown. The material properties pertinent to this study include work function, emissivity, thermal conductivity, and specific heat and are summarized in Table 2. Previous studies have shown that ETC is more effective for higher emitted electron currents from the surface, which can occur with a lower function material. For that reason, a low work function of $2 \mathrm{eV}$ (e.g. value for Cesium $^{22}$ ) will be used in the test cases. Material emissivity impacts how effective radiative cooling is, with a blackbody having the most effective radiative cooling. Although previous studies have shown that ETC is more effective for lower material emissivities, leading edge surfaces of hypersonic vehicles are typically close to blackbody so the material emissivity will be assumed to equal 1.0. Thermal conductivity for TPS materials are typically anisotropic and temperature dependent. In order to clarify the analysis, a low and high thermal conductivity typical of a TPS material were chosen and assumed to be temperature independent and isotropic in the test cases. Titanium can have as low as thermal conductivity as approximately $20 \mathrm{~W} / \mathrm{m} \mathrm{K}^{23}$ while UHTCs have a larger thermal conductivity of approximately $80 \mathrm{~W} / \mathrm{m} \mathrm{K} .{ }^{24}$ A low and high value was also chosen for specific heat and also assumed to be temperature independent. UHTCs and titanium both have a specific heat capacities of approximately $600 \mathrm{~J} / \mathrm{kg} \mathrm{K}$ but a larger value was also chosen to investigate the effect of specific heat capacity. It is to be noted that the potential bias of the surface (or virtual cathode if emission is space-charge limited) is assumed to equal $-5 \mathrm{~V}$.

Table 2: Material properties

\begin{tabular}{|c|c|c|c|c|}
\hline $\begin{array}{c}\text { Work Function, } \\
\text { eV }\end{array}$ & Emissivity & & $\begin{array}{l}\text { nductivity, } \\
\mathrm{m} \mathrm{K}\end{array}$ & $\begin{array}{c}\text { Specific Heat Capacity, } \\
\text { J / kg K }\end{array}$ \\
\hline 2.0 & 1.0 & 20 & 80 & 1200 \\
\hline
\end{tabular}

\section{B. In-depth Material Conduction}

Simulations were done using MOPAR for the conditions of interest mentioned in Tables 1 and 2 without ETC implemented. The purpose of the simulations were to assess the effect in-depth surface conduction has on surface temperature and determine typical in-depth surface heat transfer profiles for the test cases of interest. Figure 3 presents the in-depth surface conduction heat transfer profiles for different thermal conductivities, heat capacities, and durations of flight. For both thermal conductivities, the shortest durations result in the highest in-depth heat transfer. This is due to the limited time for the heat to conduct into the material, resulting in large temperature gradients within the material driving the higher heat flux due to Fourier's law. As the duration of the flight is lengthened, the surface conduction heat flux is reduced. The conduction reaches steady-state before five minutes of flight time as shown by the heat transfer staying steady between five and ten minutes. As expected, a higher thermal conductivity results in a higher surface conduction as shown between Figs. 3a and 3b. The effect of the heat capacity is only shown before the material reaches steady-state, which is that a higher heat capacity requires more energy to heat up the material, so the larger temperature gradients persist in the material longer resulting in higher surface conductions. The resulting surface temperatures are shown in Fig. 4. The longer durations of flight result in a larger 
surface temperature, as expected. The steady-state (5 and 10 minute durations) cases with a lower thermal conductivity result in a higher surface temperature than the cases with a high thermal conductivity. This is expected because with less of the heat conducted into the material, a higher surface temperature is required to radiate more of the heat back into the flow. This is illustrated by Fig. 5, which shows what percentage of the heat away from the surface is from radiation and in-depth conduction. For the low thermal conductivity, radiative cooling contributes more to heat away form the surface than in-depth surface conduction for each case besides the short-duration with a high specific heat. For the high thermal conductivity, at least near the stagnation point, in-depth surface conduction contributes more of the heat transfer away from the surface than radiative cooling.

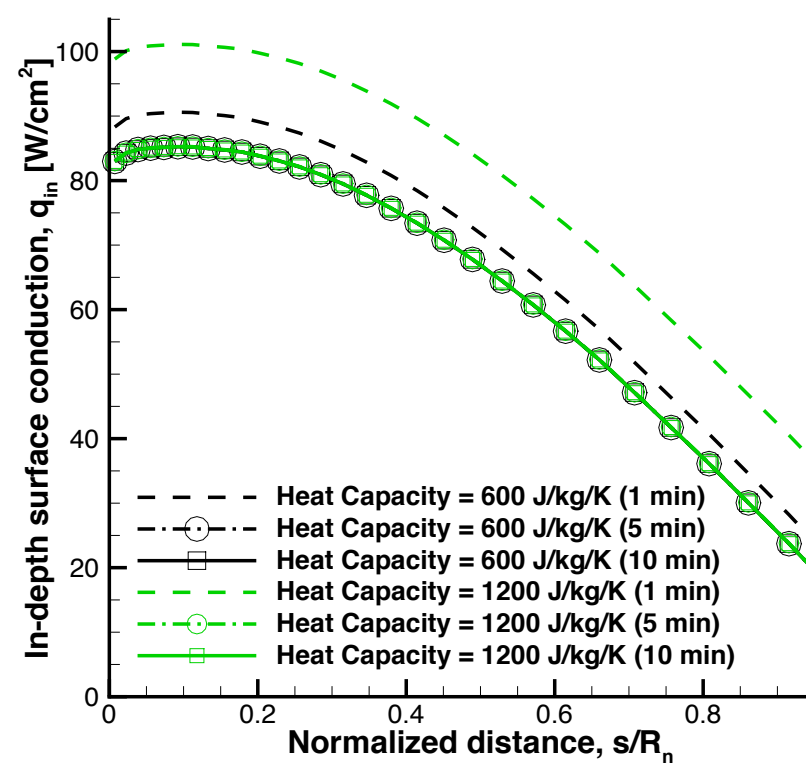

(a) Thermal conductivity $=20 \mathrm{~W} / \mathrm{m} \mathrm{K}$

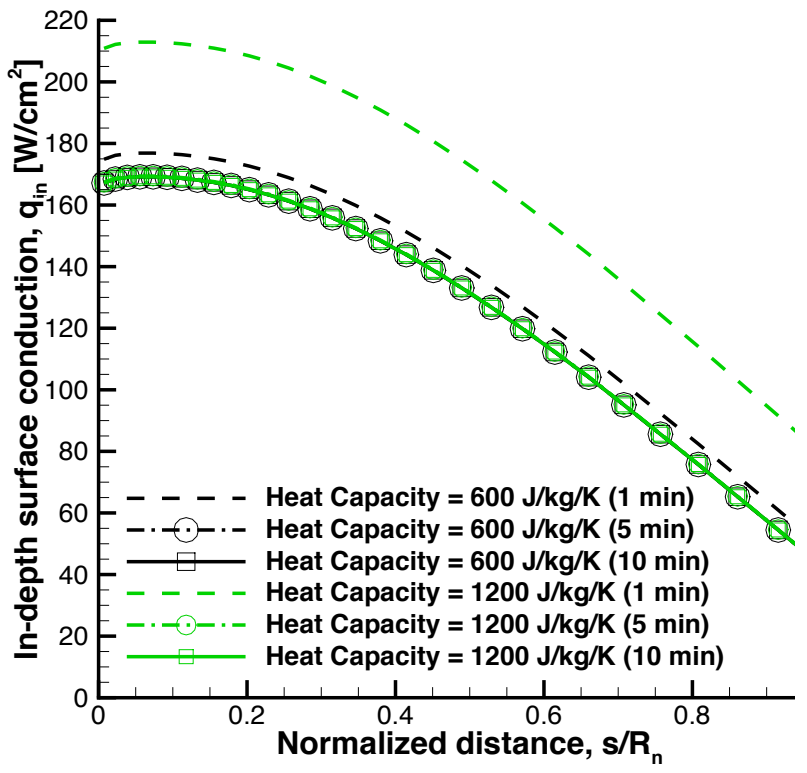

(b) Thermal conductivity $=80 \mathrm{~W} / \mathrm{m} \mathrm{K}$

Figure 3: In-depth surface conduction profiles for $6 \mathrm{~km} / \mathrm{s}$ case

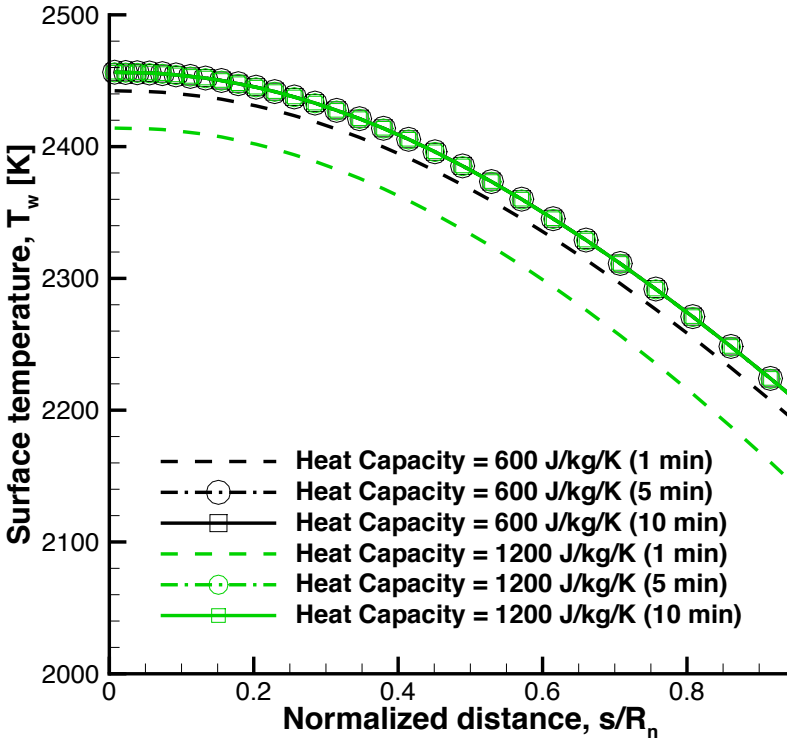

(a) Thermal conductivity $=20 \mathrm{~W} / \mathrm{m} \mathrm{K}$

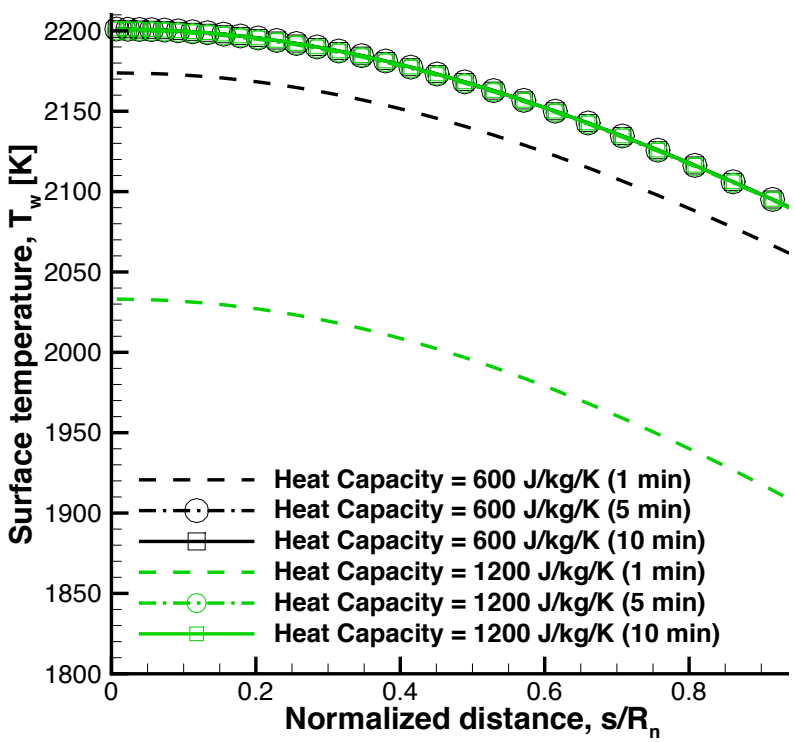

(b) Thermal conductivity $=80 \mathrm{~W} / \mathrm{m} \mathrm{K}$

Figure 4: Surface temperature profiles for $6 \mathrm{~km} / \mathrm{s}$ case 


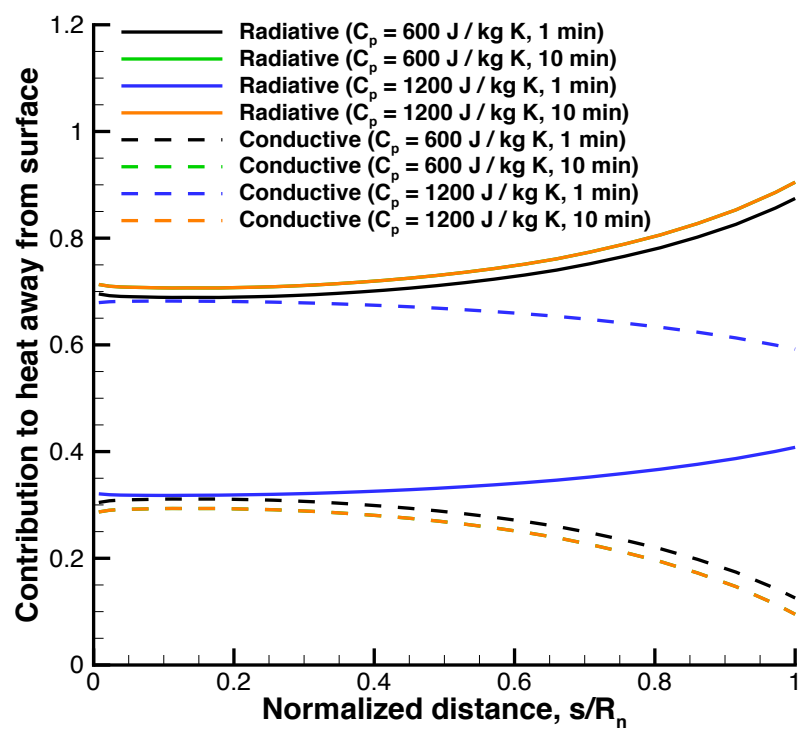

(a) Thermal conductivity $=20 \mathrm{~W} / \mathrm{m} \mathrm{K}$

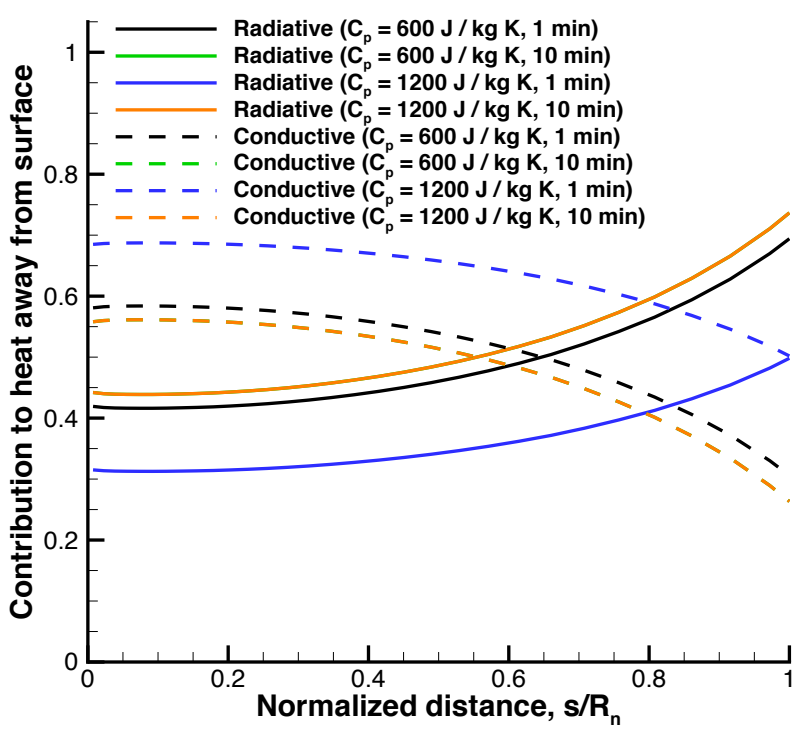

(b) Thermal conductivity $=80 \mathrm{~W} / \mathrm{m} \mathrm{K}$

Figure 5: Contribution to heat away from the surface for $6 \mathrm{~km} / \mathrm{s}$ case

For the cases with ETC, another mode of heat transfer away from the surface will be present complementing radiative cooling and in-depth surface conduction. Since ETC carries energy into the flow and away from the surface, it effectively reduces the convective heating rate the material experiences. In order to simulate the effect of in-depth surface conduction when the effective convective heat transfer is smaller, a smaller freestream velocity of $4 \mathrm{~km} / \mathrm{s}$ was evaluated with the resulting in-depth surface conduction profiles shown in Fig. 6. Although the convective heating rate is smaller for the slower velocity, the in-depth surface conduction values are comparable to the $6 \mathrm{~km} / \mathrm{s}$ case.

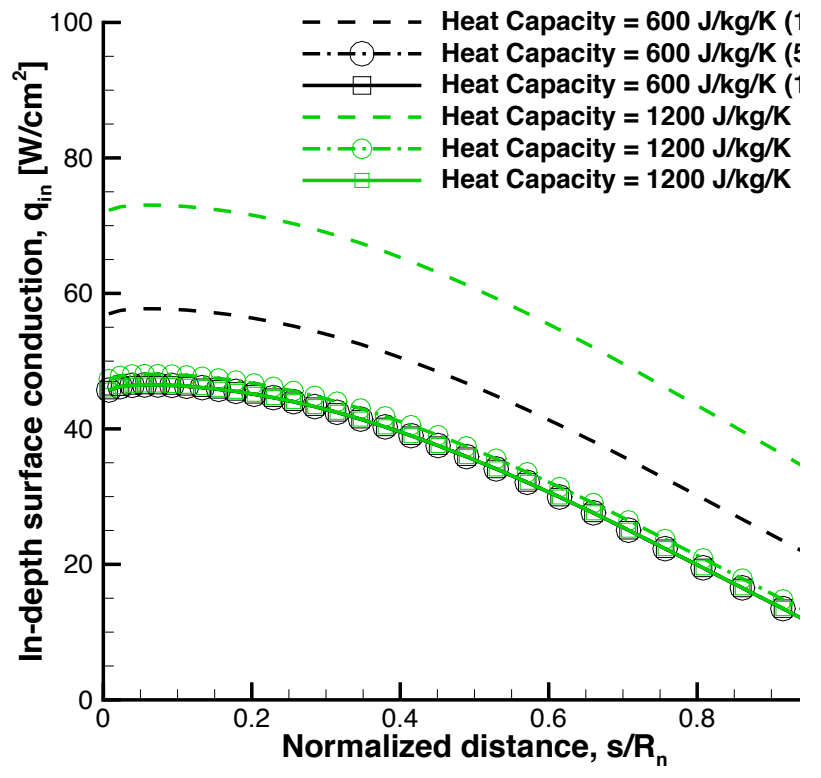

(a) Thermal conductivity $=20 \mathrm{~W} / \mathrm{m} \mathrm{K}$

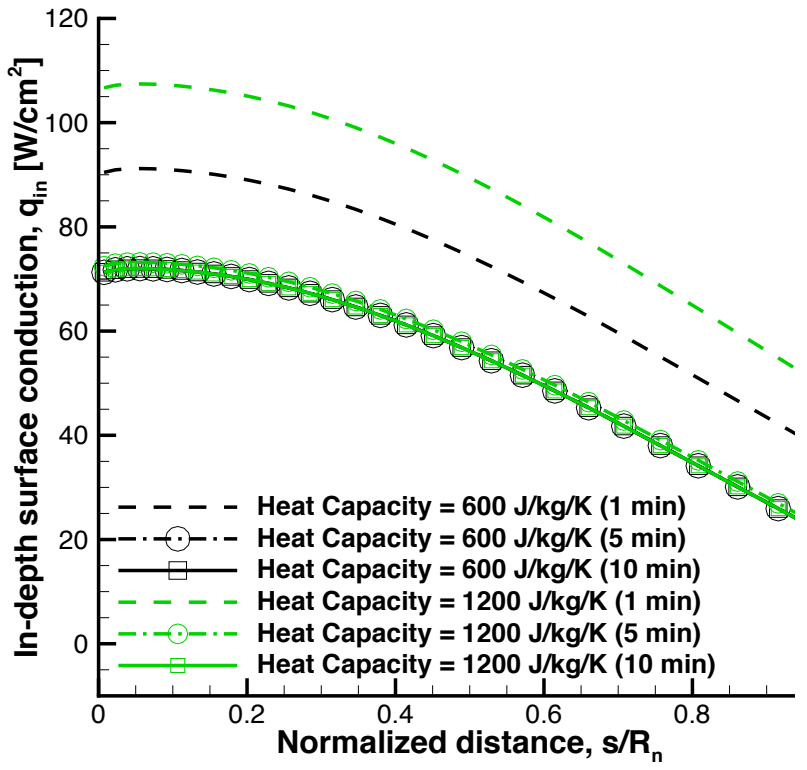

(b) Thermal conductivity $=80 \mathrm{~W} / \mathrm{m} \mathrm{K}$

Figure 6: In-depth surface conduction profiles for $4 \mathrm{~km} / \mathrm{s}$ case 


\section{Effect of In-depth Material Conduction on ETC}

Using profiles from the MOPAR cases, three different peak in-depth conduction values were chosen: 50, 100, and $200 \mathrm{~W} / \mathrm{cm}^{2}$. Profiles were then generated using by scaling the profiles in the MOPAR study and are shown in Fig. 7. These profiles were implemented in the ETC framework in order to investigate the effect in-depth surface conduction has on ETC. The resulting surface temperature profiles are shown in Fig. 8. Without ETC and in-depth conduction, the surface temperature is the highest, as expected, because the only mode of heat away from the surface is radiation. For the ideal ETC case, where the level of emission is determined only by Eq. 8 and neglecting space-charge effects with no in-depth conduction, the surface temperature is reduced by $40 \%$ at the stagnation point. If space-charge effects are accounted for but still no in-depth surface conduction is modeled, the reduction of surface temperature at the stagnation point is reduced by $17 \%$. For the same case but with in-depth conduction heat transfer value of 50, 100, and 200 $\mathrm{W} / \mathrm{cm}^{2}$, the surface temperature at the stagnation point is reduced by $21 \%, 29 \%$, and $45 \%$, respectively. All of the cases with in-depth surface conduction and space-charge limited emission result in a lower stagnation point surface temperature than the space-space limited emission alone, and one value actually is lower than the ideal emission without conduction. The wiggles in the cases with in-depth surface conduction are due to the set in-depth surface conduction profiles only occurring near the stagnation point, so the surface temperatures converge back to the case without conduction further along the body. A smooth curve would be expected if MOPAR was coupled to the ETC framework and a predetermined conduction profile was not used (e.g. fully-coupled heat transfer balance). The heat transfer balance, which determines the surface temperature, is an intricate balance with the space-charge limited ETC being heavily dependent on the level of ionization of the flow at the surface, ${ }^{7}$ so oscillating surface temperatures are expected when using an approximate profile for in-depth surface conduction. The contributions to the heat leaving the surface are shown in Fig. 9. For the case with ideal ETC and no in-depth surface conduction, the heat away from the surface is dominated by ETC heat transfer. For the case with space-charge limited ETC, the contribution from ETC is smaller but still more than radiative cooling. For the cases with 50 and 100 $\mathrm{W} / \mathrm{cm}^{2}$ surface conductions, the ETC contribution is similar to that of the case without conduction and still the dominant mode of heat away from the surface. However, when the conduction is $200 \mathrm{~W} / \mathrm{cm}^{2}$, in-depth surface conduction becomes the dominant mode of heat away from the surface. For each case, radiative cooling is the least dominant mode of heat away from the surface.

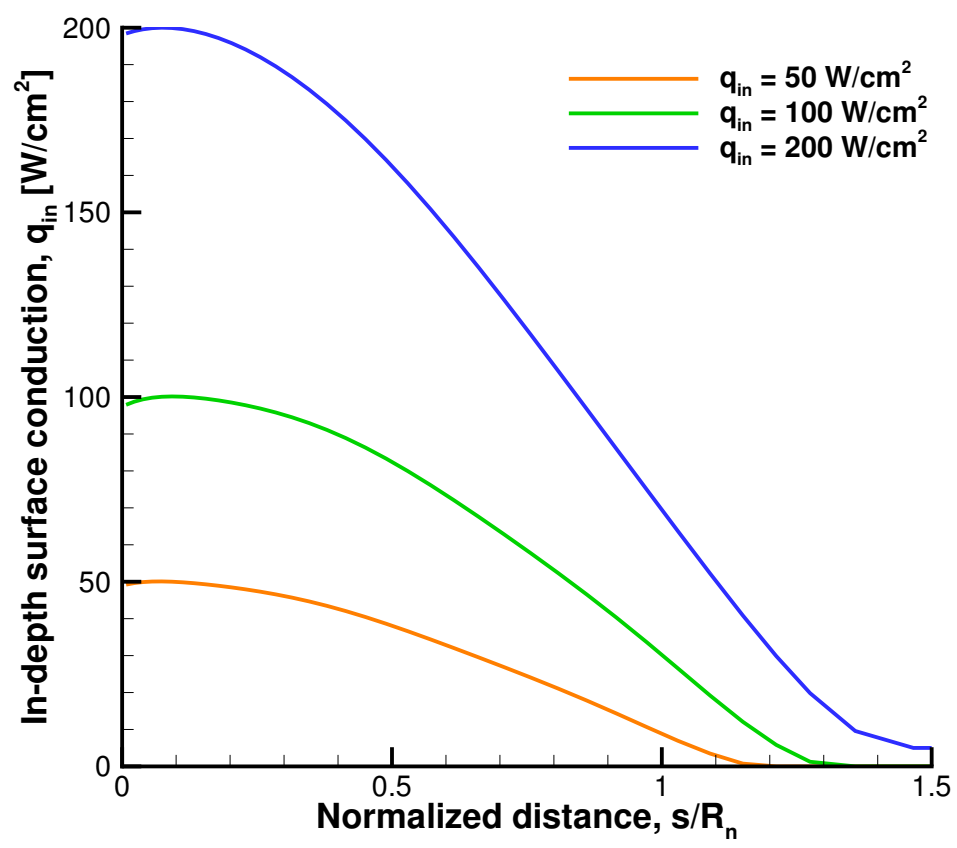

Figure 7: Manufactured in-depth surface conduction profiles used for ETC study 


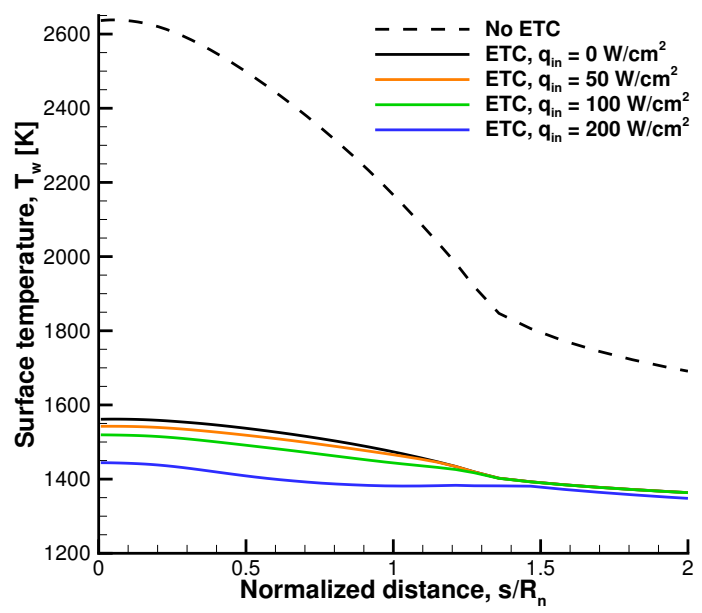

(a) Ideal emission

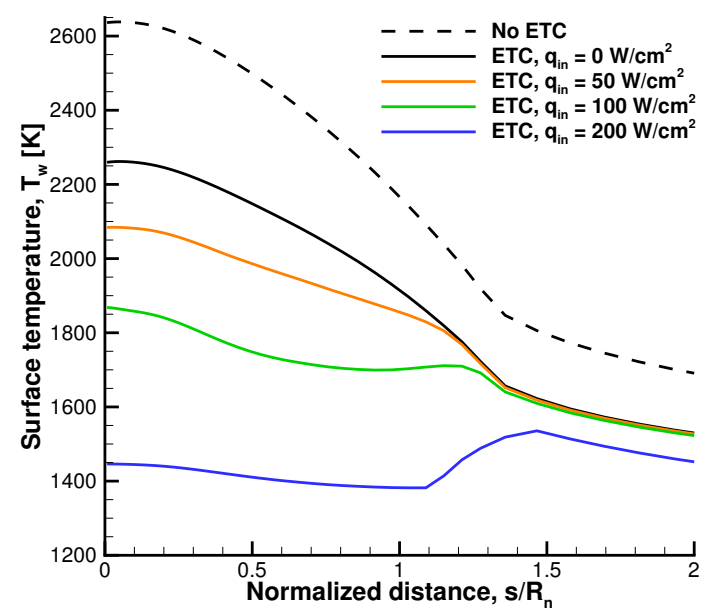

(b) Space-charge-limited emission

Figure 8: Surface temperature profiles for $6 \mathrm{~km} / \mathrm{s}$ case with in-depth conduction and ETC

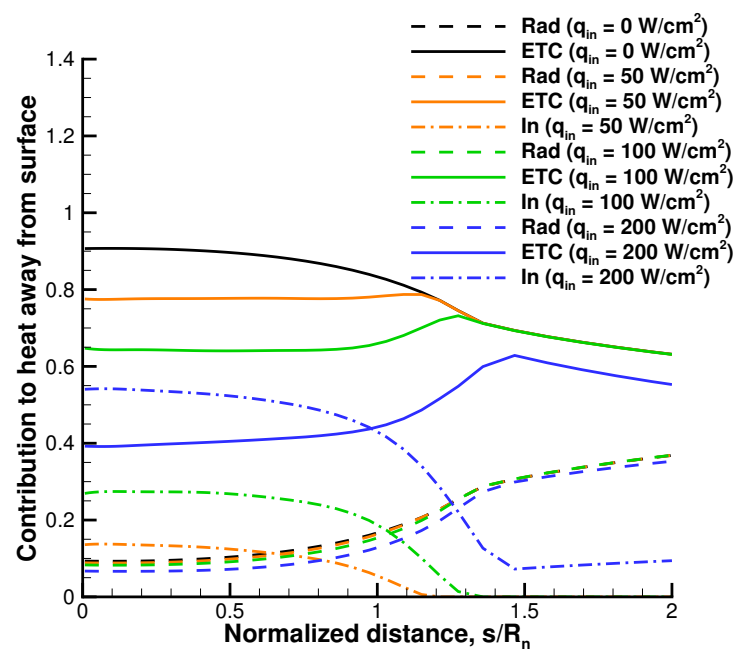

(a) Ideal emission

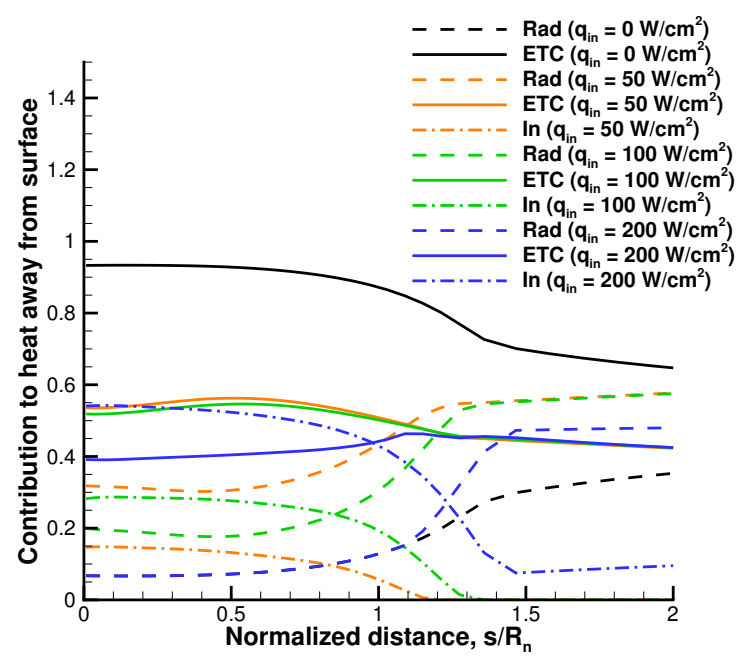

(b) Space-charge-limited emission

Figure 9: Contributions to heat away from surface for $6 \mathrm{~km} / \mathrm{s}$ case with in-depth conduction and ETC

\section{Conclusions and Future Work}

The goal of the present work was to assess the effect of in-depth heat conduction has on electron transpiration cooling (ETC). For the test cases considered, typical in-depth surface conduction profiles were generated based on a parametric study using a material response code in order to estimate the levels of in-depth surface conduction hypersonic vehicles could experience. The in-depth surface profiles were then implemented into the ETC framework to investigate the effect in-depth surface conduction has on the surface properties, specifically surface temperature. The study showed that including in-depth surface conduction in the surface heat balance results in lower surface temperatures than predicted with ETC and radiative cooling alone. This is expected because in-depth surface conduction complements ETC and radiative cooling as another mode of heat transfer away from the surface, albeit into the material. The study also showed that 
ETC remains a major mode of heat transfer away from the surface, even with in-depth surface conduction. This suggests that ETC is still a promising mode of thermal management, especially since ETC transfers energy to the flow instead of into the material.

In order to continue to improve the modeling capabilities of ETC and to analyze it's feasibility as a viable option for thermal management, the analysis has to not only look at the leading edge but also at a larger scale. This includes tracking the electrons in the flowfield back to the vehicle in order to ensure that the vehicle does not charge. Also, experiments are needed to continue to validate the numerical approaches. Finally, a more accurate assessment of the effect of in-depth surface conduction would include the material response code being coupled to the ETC analysis. A fully-coupled approach would also allow for ETC to be investigated for flight trajectories instead of only steady-state.

\section{References}

${ }^{1}$ Anderson, J. D., Hypersonic and High-Temperature Gas Dynamics, 2nd ed., AIAA, Reston, VA, 2006.

${ }^{2}$ Lees, L. and Kubota, T., "Inviscid Hypersonic Flow Over Blunt-Nosed Slender Bodies," Journal of the Aeronautical Sciences, 1957, pp. 195-202.

${ }^{3}$ Fay, J. A. and Riddell, F. R., "Theory of Stagnation Point Heat Transfer in Dissociated Air," Journal of the Aeronautics Sciences, Vol. 25, 1958, pp. 73-85.

${ }^{4}$ Glass, D. E., "Ceramic Matrix Composite (CMC) Thermal Protection Systems (TPS) and Hot Structures for Hypersonic Vehicles," AIAA Paper 2008-2682, 2008.

${ }^{5}$ Voland, R. T., Huebner, L. D., and McClinton, C. R., "X-43A Hypersonic Vehicle Technology Development," Acta Astronautica, Vol. 57, 2005, pp. 614-622.

${ }^{6}$ Uribarri, L. A. and Allen, E. H., "Electron Transpiration Cooling for Hot Aerospace Surfaces," AIAA Paper 2015-3674, July 2015.

${ }^{7}$ Hanquist, K. M., Hara, K., and Boyd, I. D., "Detailed Modeling of Electron Emission for Transpiration Cooling of Hypersonic Vehicles," Journal of Applied Physics, under review, 2016.

${ }^{8}$ Bertin, J. J., "Stagnation-Region Flowfield," Hypersonic Aerothermodynamics, AIAA, Washington, DC, 1994, pp. 231-272.

${ }^{9}$ Martin, A., Scalabrin, L. C., and Boyd, I. D., "High Performance Modeling of Atmospheric Re-Entry Vehicles," Journal of Physics: Conference Series, Vol. 341, No. 1, 2012, Article 012002.

${ }^{10}$ Scalabrin, L. C., Numerical Simulation of Weakly Ionized Hypersonic Flow over Reentry Capsules, Ph. D. Dissertation, University of Michigan, Ann Arbor, 2007.

${ }^{11}$ Richardson, O. W., The Emission of Electricity from Hot Bodies, Longmans, Green and Co., 1921.

${ }^{12}$ Scott, C. D., "Wall Catalytic Recombination and Boundary Conditions in Nonequilibrium Hypersonic Flows - with Applications," Advances in Hypersonics - Modeling Hypersonic Flows, Vol. 2, 1992, pp. 176-250.

${ }^{13}$ Lieberman, M. A., and Lichtenberg, A. J., Principles of Plasma Discharges and Materials Processing, Wiley, New York, 1994, pp. 25-30.

14 Razier, Y. P., Gas Discharge Physics, Springer-Verlag, 1991.

${ }^{15}$ Fridman, A. and Kennedy, L. A., Plasma Physics and Engineering, Taylor \& Francis, New York, 2004.

${ }^{16}$ Alkandry, H., Hanquist, K. M., and Boyd, I. D., "Conceptual Analysis of Electron Transpiration Cooling for the Leading Edges of Hypersonic Vehicles," AIAA Paper 2014-2674, 2014.

17 Takamura, S., Ohno, N., Ye, M. Y., and Kuwabara, T., "Space-Charge Limited Current from Plasma-Facing Material Surface," Contributions to Plasma Physics, Vol. 44, No. 1-3, 2004, pp. 126-137.

${ }^{18}$ Wiebenga, J. E., High-Fidelity Material Response Modeling as Part of an Aerothermoelastic Framework for Hypersonic Flows, Ph. D. Dissertation, University of Michigan, Ann Arbor, MI, 2014.

${ }^{19}$ Wiebenga, J. E., "Computation of Multi-Dimensional Material Response Coupled to Hypersonic Flow," AIAA Paper 2012-2873, June 2012.

${ }^{20}$ Kuntz, D. W., Hassan, B., and Potter, D. L., "Predictions of Ablating Hypersonic Vehicles Using an Iterative Coupled Fluid/Thermal Approach," Journal of Thermophysics and Heat Transfer, Vol. 15, No. 2, 2001, pp. 129-139.

${ }^{21}$ Hanquist, K. M., Hara, K., and Boyd, I. D., "Modeling of Electron Transpiration Cooling for Hypersonic Vehicles," AIAA Paper 2016-4433, 2016.

${ }^{22}$ Haynes, W. M., CRC Handbook of Chemistry and Physics, CRC Press, Boca Raton, FL, 2013.

${ }^{23}$ Incropera, F. P., DeWitt, D. P., Bergman, T. L., and Lavine, A. S., Fundamentals of Heat and Mass Transfer, 6 ed., John Wiley \& Sons, Hoboken, 2007.

${ }^{24}$ Zimmermann, J. W., Hilmas, G. E., and Fahrenholtz, W. G., "Thermal Shock Resistance of $\mathrm{ZrB}_{2}$ and $\mathrm{ZrB}_{2}-30 \% \mathrm{SiC}$, Materials Chemistry and Physics, Vol. 112, pp. 140-145. 